\title{
Foot and ankle ligament morphometry
}

\author{
Chimba Mkandawire, PhD; ${ }^{1-2}$ William R. Ledoux, PhD; ${ }^{1,3-4}$ Bruce J. Sangeorzan, MD; ${ }^{1,3}$ Randal P. Ching, PhD ${ }^{1,3-4 *}$ \\ ${ }^{1}$ Department of Veterans Affairs Puget Sound Health Care System, Rehabilitation Research and Development Center \\ of Excellence for Limb Loss Prevention and Prosthetic Engineering, Seattle, WA; Departments of ${ }^{2}$ Bioengineering, \\ ${ }^{3}$ Orthopaedics and Sports Medicine, and ${ }^{4}$ Mechanical Engineering, University of Washington, Seattle, WA
}

\begin{abstract}
This study primarily measured and established a morphometric spectrum of cross-sectional areas for foot and ankle ligaments with the use of a new freeze-fracture technique that was independent of ligament cross-sectional shape. Ligament morphometry measurements were made on a total of 121 bone-ligament-bone preparations that were harvested from 26 fresh (unembalmed) male cadaver feet. We used the traditional digital caliper method to measure length and the freeze-fracture technique to measure cross-sectional area. Cross-sectional area values ranged from 21.36 to $170.48 \mathrm{~mm}^{2}$ and length values from 5.01 to $37.45 \mathrm{~mm}$. In addition, area-to-length ratios ranged from 0.60 to $27.02 \mathrm{~mm}$. Compared with the freeze-fracture technique, the digital caliper method, which assumed that ligament crosssectional shape was rectangular, resulted in an approximately $35 \%$ difference in cross-sectional area.
\end{abstract}

Key words: ankle, area, biomechanics, cadaver, calipers, foot, freeze-fracture, ligaments, measurement, models.

\section{INTRODUCTION}

Several three-dimensional (3-D) models of the foot, including some that use the finite element (FE) method, have recently been developed for the study of foot mechanics [1-4]. We have previously reported on a 3-D FE model of the adult foot and ankle that is being developed to investigate the disease processes associated with foot deformity [5-7]. Our and other groups' modeling efforts will provide insight on otherwise unquantifiable variables (e.g., bony motion due to muscle imbalances) and enable parametric analyses for examination and optimization of treatment strategies for clinicians. Our model used computed tomography scans to generate a total of 28 bones that were reconstructed and interconnected by 51 ligaments. The origins and insertions of these ligaments were based on anatomical texts. However, since data on mechanical testing of foot ligaments is minimal, we used linear material properties that were largely extrapolated from Siegler et al.'s work on ankle ligaments [8]. Extrapolations were based on area-to-length ratios (when available) or inverse length ratios of the ligaments. The methods for attaining ligament mechanical properties in our model and others were imprecise and did not account for features specific to the ligament morphometry of the foot (e.g., concave shapes, nonellipsoid shapes) [1-3,5-7]. Given the dearth of known material properties for foot and ankle ligaments, our goal was to collect ligament morphometry data from the foot and ankle.

\footnotetext{
Abbreviations: 3-D = three-dimensional, ACL = anterior cruciate ligament, $\mathrm{FE}=$ finite element, $\mathrm{MCL}=$ medial collateral ligament, $\mathrm{NIH}=$ National Institutes of Health, PCL = posterior cruciate ligament, PMMA = polymethylmethacrylate, $\mathrm{SD}=$ standard deviation.

This material was based on work supported by the Department of Veterans Affairs (VA), Rehabilitation Research and Development Service, grant A0806C; the University of Washington Department of Bioengineering; The Graduate School of University of Washington; and a VA Pre-Doctoral Associated Health Rehabilitation Research Fellowship.

*Address all correspondence to Randal P. Ching, PhD; Applied Biomechanics Laboratory, 501 Eastlake Avenue E, Suite 102, Seattle, WA 98109; 206-625-0756; fax: 206-625-0857. Email: rc@u.washington.edu
}

DOI: 10.1682/JRRD.2004.08.0094 
Foot and ankle ligament morphometry has not been well documented in the literature, with only ankle ligament data presented. Our studies have shown that, when compared with ligaments in the foot, ankle ligaments tend to follow a morphometry pattern: relatively long ligament lengths, similar cross-sectional shapes, and large crosssectional areas [9-10]. Siegler et al. measured mean crosssectional area and mean length for seven ankle ligaments under a constant loading condition [8]. Area measurements were performed by placement of ankle ligaments under a $22 \mathrm{~N}$ tensile load and $0.345 \mathrm{MPa}$ of compressive transverse clamping pressure (micrometer), whereas length measurements were performed separately by placement of ligaments under a $22 \mathrm{~N}$ tensile load without the applied clamping pressure [8]. Except for the work of Siegler et al. and the National Institutes of Health (NIH) Visible Human Project ${ }^{\circledR}$ (National Library of Medicine, Bethesda, Maryland), we know of no published foot and ankle ligament morphometry [8-10].

Our group has used two techniques to acquire foot and ankle ligament morphometry. First, we pursued in situ imaging modalities to measure ligament morphometry but had difficulty ascertaining ligament insertions, origins, and boundaries with magnetic resonance imaging, which led to imprecise estimations of length and cross-sectional area. Second, we successfully used the NIH Visible Human Project ${ }^{\circledR}$ work to determine ligament insertions, origins, and boundaries; however, the sample size $(n=1)$ was a limiting factor [9-10]. Direct measurement techniques provide accurate ligament lengths and cross-sectional areas. However, in situ data via direct measurement is very difficult to obtain since it typically requires that bone-ligamentbone specimens be prepared (i.e., the joint destroyed). In addition, once harvested, what the baseline length (zerostrain state) of the ligament would have been in its neutral position (i.e., was the ligament pretensioned?) is unknown.

Two predominant methods for measurement of ligament length are video analysis and digital calipers. Although video analysis has had reported accuracies of up to 99.5 percent [11], it has very high cost and time factors. Conversely, digital calipers offer ease of use with reasonable accuracy. Siegler et al. [8], Torzilli and Arnoczky [12], and Danto and Woo [13] performed ligament-length measurements with digital calipers under various applied tare loads to maintain constant tensile force or stress.

Direct cross-sectional area measurements of ligament tissue have been made with caliper and/or micrometer, laser micrometer, and casting techniques. Standard or modified calipers and/or micrometers have been used to make width and thickness measurements with the specimen subjected to a tare load and the cross-sectional area computed by assumption of a cross-sectional shape (rectangular, ellipsoid, etc.) [8,14-15]. Because ligaments do not have standard cross-sectional shapes (Figure 1), other measurement techniques have been attempted. A laser micrometer system was used for recording rabbit anterior cruciate ligament (ACL) and medial collateral ligament (MCL) cross-sectional areas [15]. Race and Amis later found that the laser micrometer system poorly approximated concave surfaces compared with a casting and photographic method for measuring posterior cruciate ligament (PCL) cross sections [16].

Du et al. performed a quick-freeze area measurement study on rat lungs. Lungs were quick-frozen and then stained and sectioned for digitization [17]. Liquid nitrogen was used as the freezing agent. To our knowledge, no one has yet used this technique to measure ligament or tendon tissue, so comparison with casting, area micrometer, ultrasonography, or laser micrometer techniques was unavailable.

The objective of this research was the measurement and establishment of a morphometric spectrum of the lengths and cross-sectional areas of foot and ankle ligaments with a technique independent of ligament cross-sectional shape. The ligament data collected would enable more reliable approximations of foot and ankle ligament properties in the absence of material property data and would also be the first step toward development of structure-property relationships for a broad spectrum of ligaments.

\section{METHODS}

This study was approved by the University of Washington Institutional Review Board. We acquired 26 lower-leg cadaver specimens (taken from 20 males, 6 who produced matching pairs and 14 who produced a left or right specimen) through the University of Washington Willed Body Program. From these feet, 20 different ligaments were harvested (Table 1) for a total of 121 bone-ligament-bone specimens. The number of ligaments harvested for each ligament type varied (Table 1). The age (mean \pm standard deviation [SD]) of specimen donors was $77.4 \pm 19.7 \mathrm{yr}$ (range 26-94 yr). Ligament nomenclature and attachment sites are referred to as described in Sarrafian [18]. Table 1 provides a key to the ligament acronyms used throughout this article. 
(a)

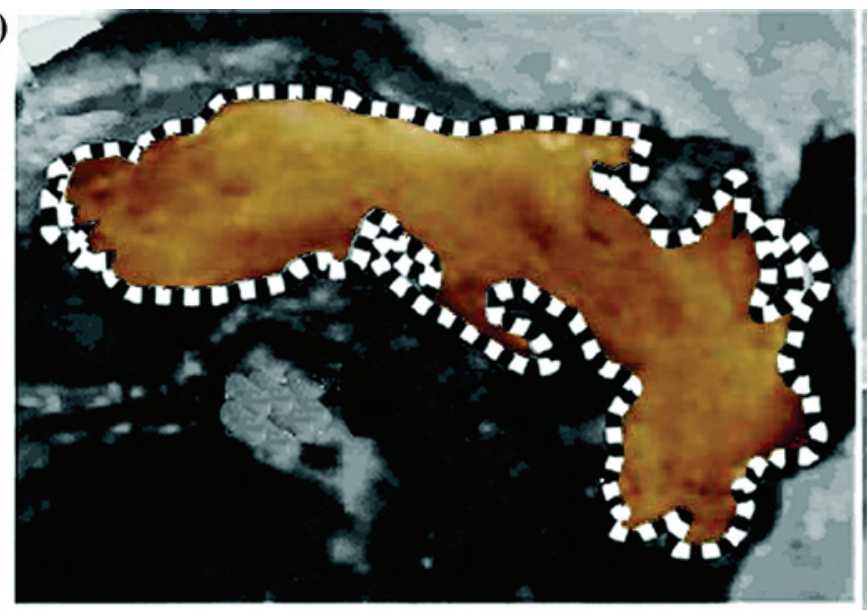

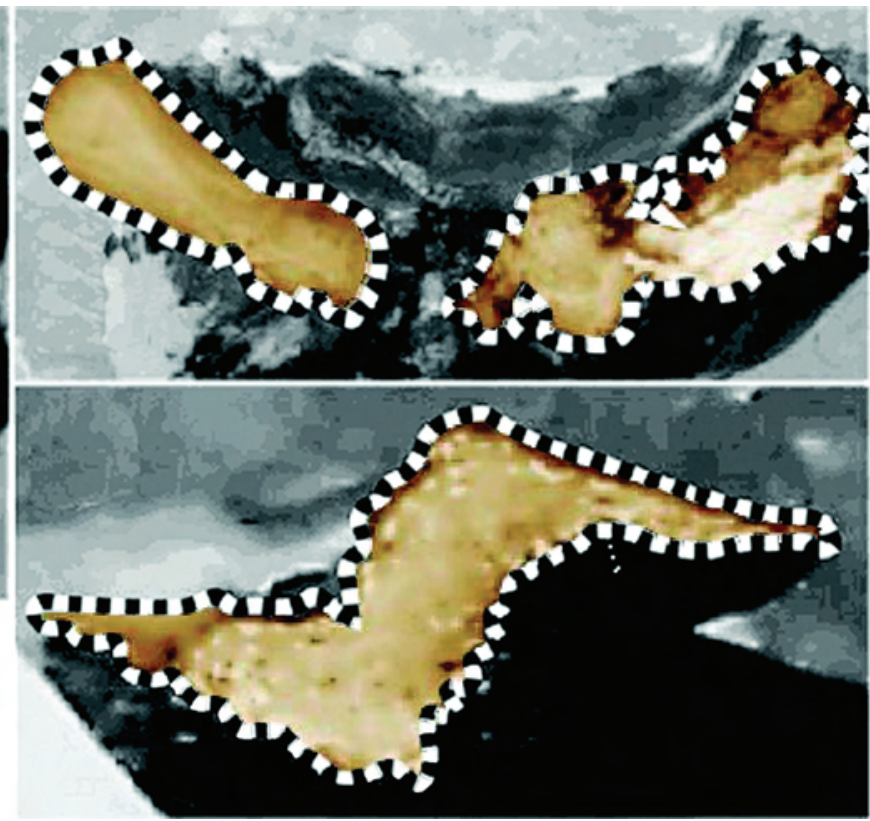

(b)

Figure 1.

Sample ligament cross-sectional shapes: (a) PC1M1, (b) ICN, and (c) ATTL. Dotted lines delineate ligament cross-sectional shape. See Table 1 for key to ligament acronyms.

Table 1.

Ligament acronym key and number tested for each ligament type.

\begin{tabular}{llc}
\hline \multicolumn{1}{c}{ Ligament } & Acronym & No. \\
\hline Anterior Talofibular Ligament & ATFL & 5 \\
Anterior Tibiotalar Ligament & ATTL & 6 \\
Calcaneofibular Ligament & CFL & 6 \\
Dorsal Cuboid Fifth-Metatarsal & DCbM5 & 6 \\
Dorsal First-Cuneiform First-Metatarsal & DC1M1 & 6 \\
Dorsal Fourth-Metatarsal Fifth-Metatarsal & DM4M5 & 5 \\
Dorsal Second-Cuneiform Second-Metatarsal & DC2M2 & 6 \\
Dorsal Second-Cuneiform Navicular & DC2Nv & 6 \\
Dorsal Third-Metatarsal Fourth-Metatarsal & DM3M4 & 6 \\
Inferior Calcaneonavicular & ICN & 7 \\
Interosseous First-Metatarsal Second-Metatarsal & IM1M2 & 6 \\
Interosseous Fourth-Metatarsal Fifth-Metatarsal & IM4M5 & 7 \\
Interosseous Second-Cuneiform Third-Cuneiform & IC2C3 & 6 \\
Interosseous Third-Cuneiform Cuboid & IC3Cb & 6 \\
Medial First-Cuneiform First-Metatarsal & MC1M1 & 6 \\
Plantar First-Cuneiform First-Metatarsal & PC1M1 & 6 \\
Posterior Talofibular Ligament & PTFL & 6 \\
Posterior Tibiotalar Ligament & PTTL & 6 \\
Short Plantar Ligament & SPL & 6 \\
Tibiocalcaneal Ligament & TCL & 6 \\
\hline \hline
\end{tabular}

As the ligaments were harvested, large bony ends were shaped with an oscillating bone saw (Model 810 Autopsy Saw, Stryker Corp, Kalamazoo, Michigan) so that each bone-ligament-bone specimen would fit into its potting cup. Once ligaments were harvested, Voerhoeff's elastin stain (VWR Scientific Products, Seattle, Washington) was used to mark by visual inspection the center of each ligament's origin and insertion (Figure 2) [11].

Ligaments were potted in polymethylmethacrylate (PMMA) (Lang Dental Manufacturing Company, Inc, Wheeling, Illinois) with the use of a stainless steel wire reinforcing procedure adapted from Bettinger et al. [19]. Several sections of $1 \mathrm{~mm}$ diameter steel wire were drilled into each bony end to increase the purchase strength between the PMMA and the bony tissue. Potting cups were made from $63.5 \mathrm{~mm}$ diameter Schedule-40 polyvinyl chloride pipe cut into $38.1 \mathrm{~mm}$ lengths. A potting apparatus was used that aligned the ligament axis perpendicular to the ground. The amount of potting material used was uniform from cup to cup, with each potting cup weighing approximately $1.5 \mathrm{~N}$ after curing.

Length measurements were performed with a set of digital calipers (Mitutoyo Series 500, Mitutoyo America Corporation Headquarters, Aurora, Illinois) by manual suspension of one potted end (Figure 2). Measurements 


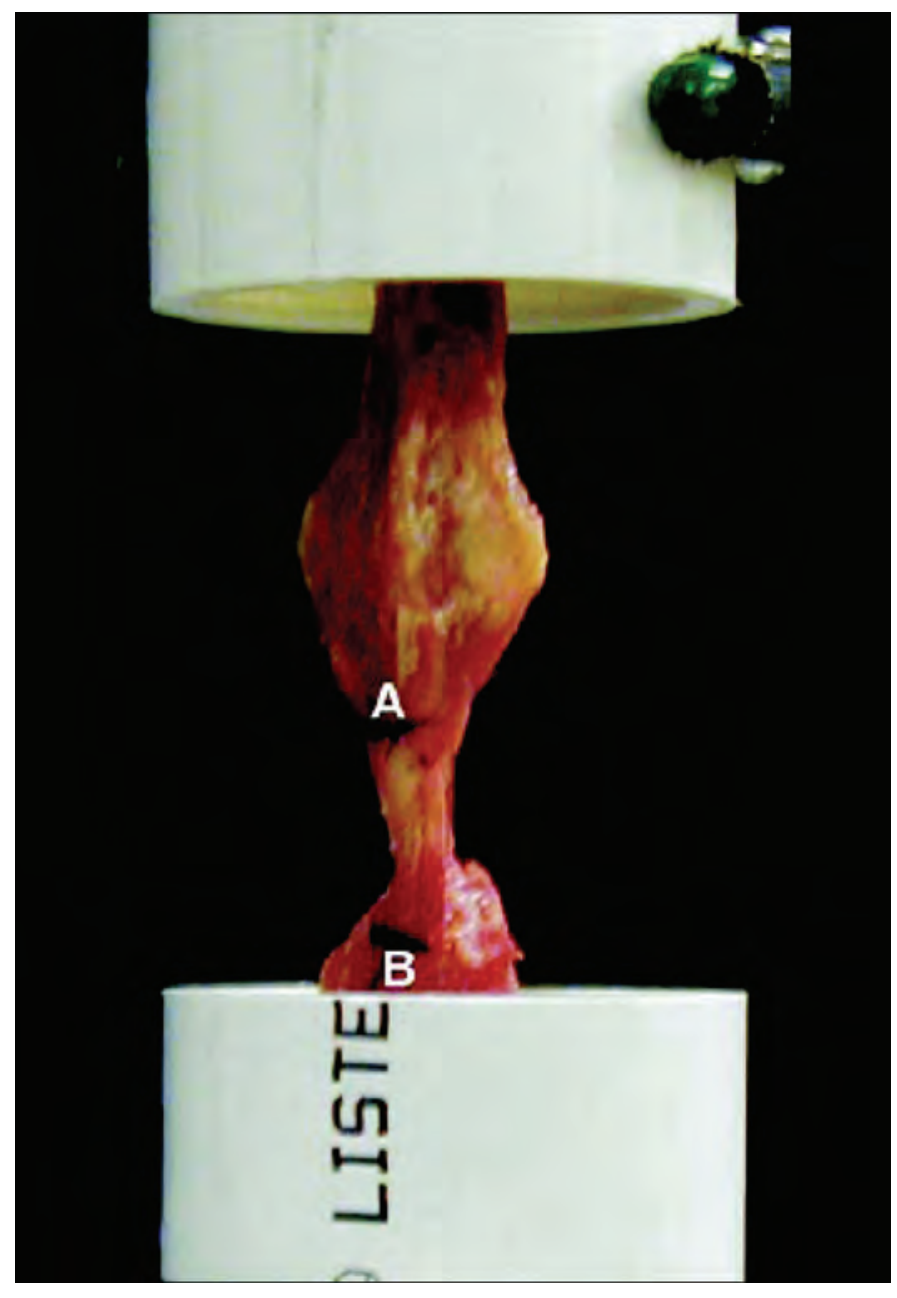

Figure 2.

Ligament suspension for caliper measurement. Voerhoeff's elastin stain marks for (A) fibula attachment and (B) center of calcaneus attachment for calcaneofibular ligament. Ligament length was determined by digital calipers that measured distance (leading to trailing edge) between $\mathrm{A}$ and $\mathrm{B}$.

were taken from the leading edge of the origin mark (labeled A in Figure 2) to the trailing edge of the insertion mark (labeled B in Figure 2). The dye was applied so that the outside edge of the dye would define the bonyligament interface. Width and thickness measurements were taken with digital calipers midway between the two marks that identified insertion and origin locations. The location for these measurements was determined by visual inspection.

Cross-sectional area was determined from the digital caliper width and thickness measurements; both a rectangular and an ellipsoid approximation were determined.
The rectangular cross-sectional area was calculated from the product of measured width and measured thickness. The ellipsoid cross-sectional area was calculated from the product of measured thickness, measured width, and $1 / 4 \pi$.

To more accurately measure the cross sections of the ligaments, we developed a freeze-fracture technique in which the ligaments were flash-frozen and then fractured so cross sections could be photographically analyzed. Each ligament was coated with a methyl blue dye to enhance contrast during imaging. Subsequently, ligaments were quick-frozen with an immersion technique [20]. Isopentane was chilled to subzero temperatures by a liquid nitrogen bath. Liquid nitrogen was constantly replenished so that a $25.4 \mathrm{~mm}$ thick layer of solid isopentane was always present. Isopentane was chosen because of its stability at subzero temperatures and inert behavior with human tissue. Potted bone-ligament-bone specimens were immersed with the ligament axis vertical for $60 \mathrm{~s}$.

Frozen ligaments were removed from the isopentane and prepared for sectioning. With a hammer and ground wood chisel, each ligament was bisected by a sharp perpendicular blow. Estimation of the location for bisection was performed by visual inspection based on the ligament insertion and origin marks. Each potting cup was manually held over a chopping block during bisection. The chisel was ground regularly to maintain a sharp edge. Removal of uneven edges from the bisection procedure was not necessary because the 2-D digital image acquisition essentially "flattens" any out-of-plane protrusions.

After being sectioned, each ligament half was inspected for dye penetration. If methyl blue penetrated beyond the surface of the newly exposed cross section and impaired the image quality, Liquid Paper correction fluid (The Gillette Company, Boston, Massachusetts) was applied to the surface of the cross section for enhanced contrast. Methyl blue was reapplied if the freeze-fracture technique induced a loss of color to the ligament boundary (perimeter) thereby diminishing its contrast.

We mounted each ligament cross section into a vice clamp to hold the ligament axis parallel to the ground (Figure 3). A 2.1 megapixel digital camera (Model DSCP50, Sony Corp, New York, New York) was mounted onto a camera stand and positioned with the aid of a level. For calibration, a circular object (a penny) of known dimensions (diameter $19.07 \pm 0.005 \mathrm{~mm}$ ) was placed in the field of view of the camera and the same plane of the sectioned ligament face when the digital image was acquired (Figures 3-4). Twenty samples of penny crosssectional area measurements were taken randomly with 


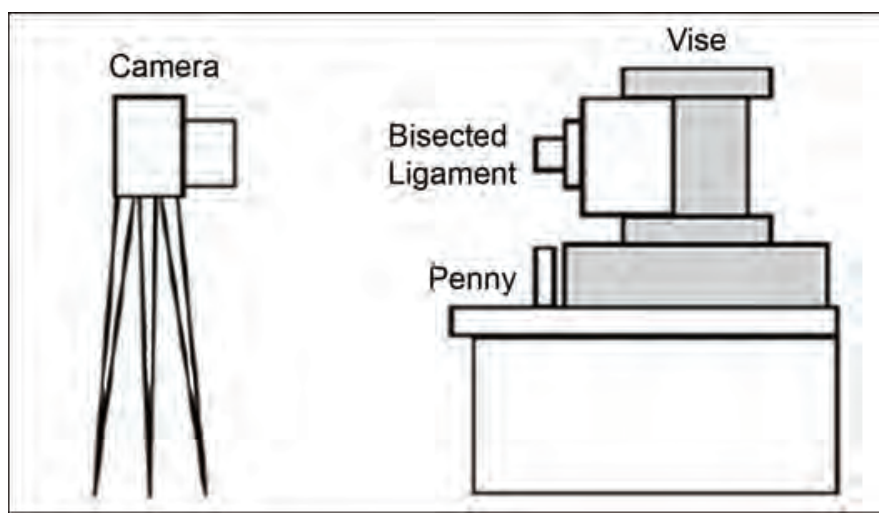

Figure 3.

Diagram of imaging setup. Face of penny used for calibration was in plane with face of bisected ligament.

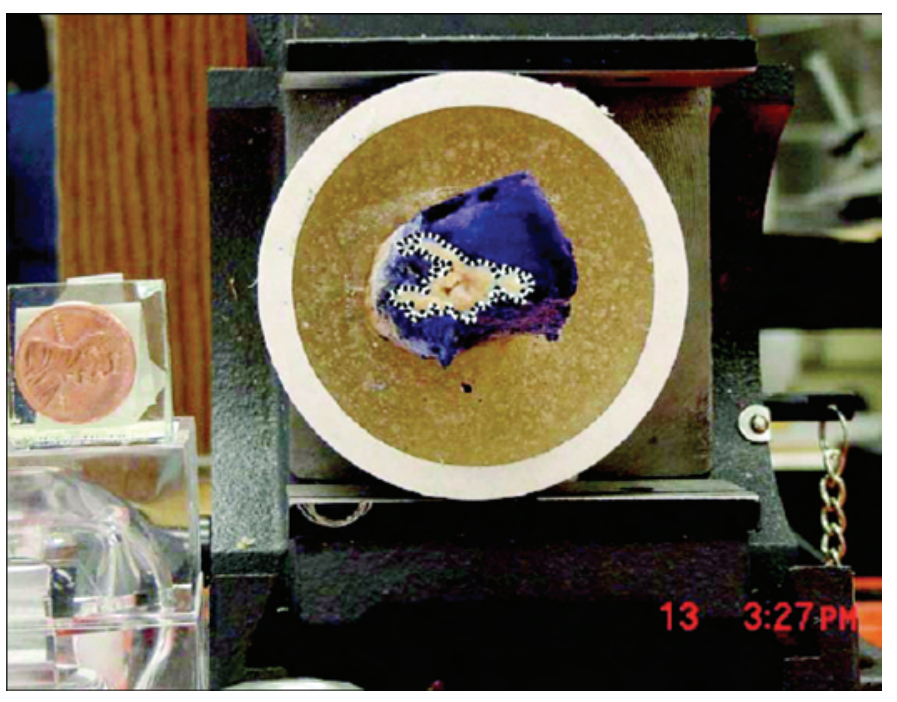

Figure 4.

Imaging setup through camera lens. Penny provided calibration data during area measurement process. Note how closely size of penny in vertical and horizontal axes approximated size of ligament. Dotted line delineates ligament cross-sectional shape.

the image analysis software described in the next paragraph and showed fluctuations of 1.8 percent difference from the area calculation $\left(\pi r^{2}\right)$ based on caliper measurements of penny diameter.

After the image was captured, area measurements were performed with a digital image analysis program (Scion Image Beta 4.0.2 Win, Scion Corporation, Frederick, Maryland). Within Scion Image, each ligament cross section was given orthogonal scales from the known orthogonal dimensions of the penny. Measurements were taken by the spanning of line segments around each ligament shape until an enclosure was generated; a built-in area function was then used to calculate the area of each enclosure.

Area measurements from the freeze-fracture and the caliper methods were compared. Two estimates of area (rectangular and ellipsoid) were used for comparison. We determined the average percent difference between the freeze-fracture and the caliper measurements by using the freeze-fracture measurements as the denominator and calculating the average from the absolute value of the difference. The following ligaments were not included because of difficulty in cross-sectional area measurement via calipers: IC2C3, IC3Cb, and IM4M5. To address whether the location of freeze-fracture measurements along the longitudinal axis of the ligament were the same location for caliper area measurements along the longitudinal axis, we compared measurements of ligament width (longest dimension) and thickness (shortest dimension) between both techniques.

\section{RESULTS}

Digital photographs of the freeze-fractured foot and ankle ligaments demonstrate nonuniform cross-sectional shapes (Figure 5). These samples of ligament bisections have had methyl blue dye added for enhanced contrast between the ligament cross section and the remaining ligament and bony insertion. Furthermore, Figures 5(a)-5(d) and 5(f) have had Liquid Paper added for enhanced contrast of the ligament cross-sectional surface.

The average area and length morphometry for each ligament type \pm 1 SD is reported in Table 2. The IC3Cb ligament had the greatest average area $\left(170.48 \mathrm{~mm}^{2}\right)$, the TCL ligament had the greatest average length $(37.45 \mathrm{~mm})$, and the IC3Cb had the greatest average area-to-length ratio (26.67 mm). The mean morphometric spectrum of the 20 different ligament types that were examined varied from 5 to $37 \mathrm{~mm}$ in length and 21 to $170 \mathrm{~mm}^{2}$ in area (Figure 6). The overall mean \pm SD area, length, and area-to-length ratio were $66.73 \pm 48.56 \mathrm{~mm}^{2}, 16.90 \pm 10.68 \mathrm{~mm}$, and $6.43 \pm 8.05 \mathrm{~mm}$, respectively.

We compared the cross-sectional area measurements estimated by the digital calipers with the freeze-fracture measurements (Table 3) by using the freeze-fracture values as the denominator for determination of percent differences. Across all 121 ligaments, the rectangular approximation had an average absolute percent difference of 35.7 percent, while the ellipsoid approximation had an average absolute percent difference of 40.0 percent. 
(a)

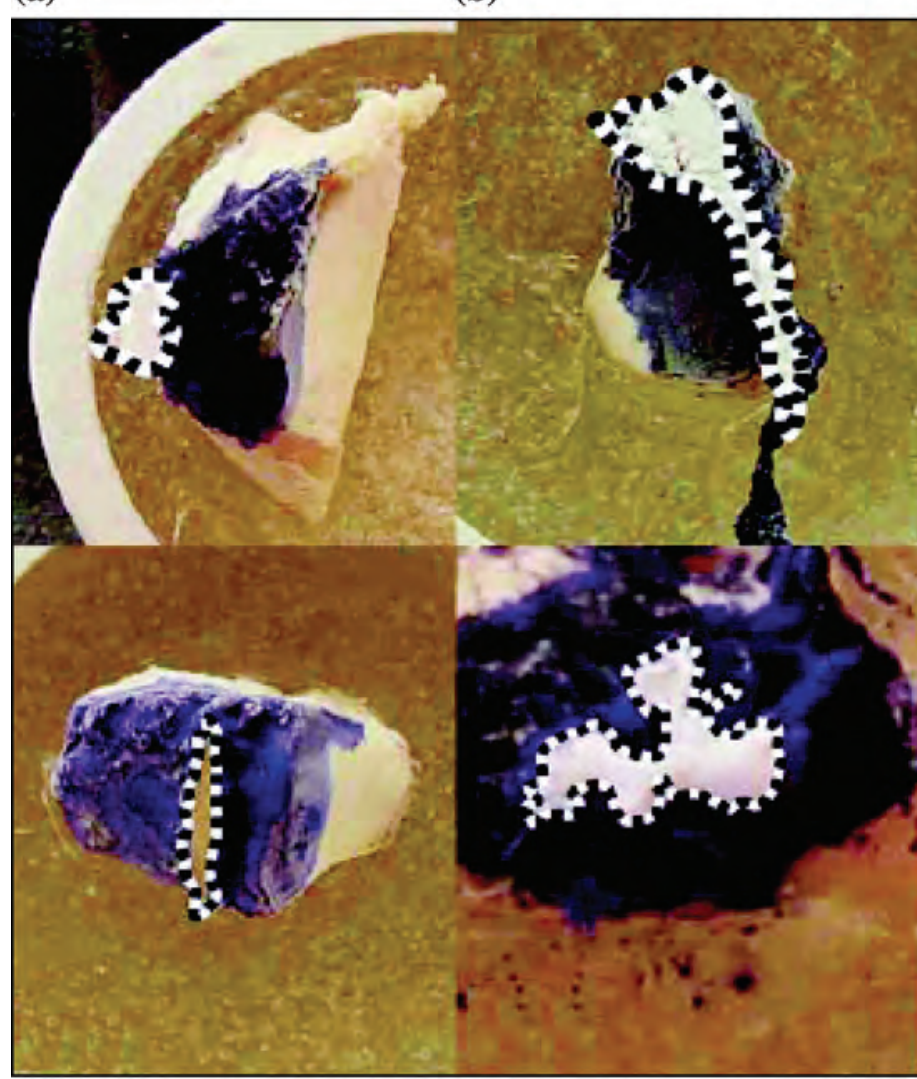

(e)

(b) (c)

(d)

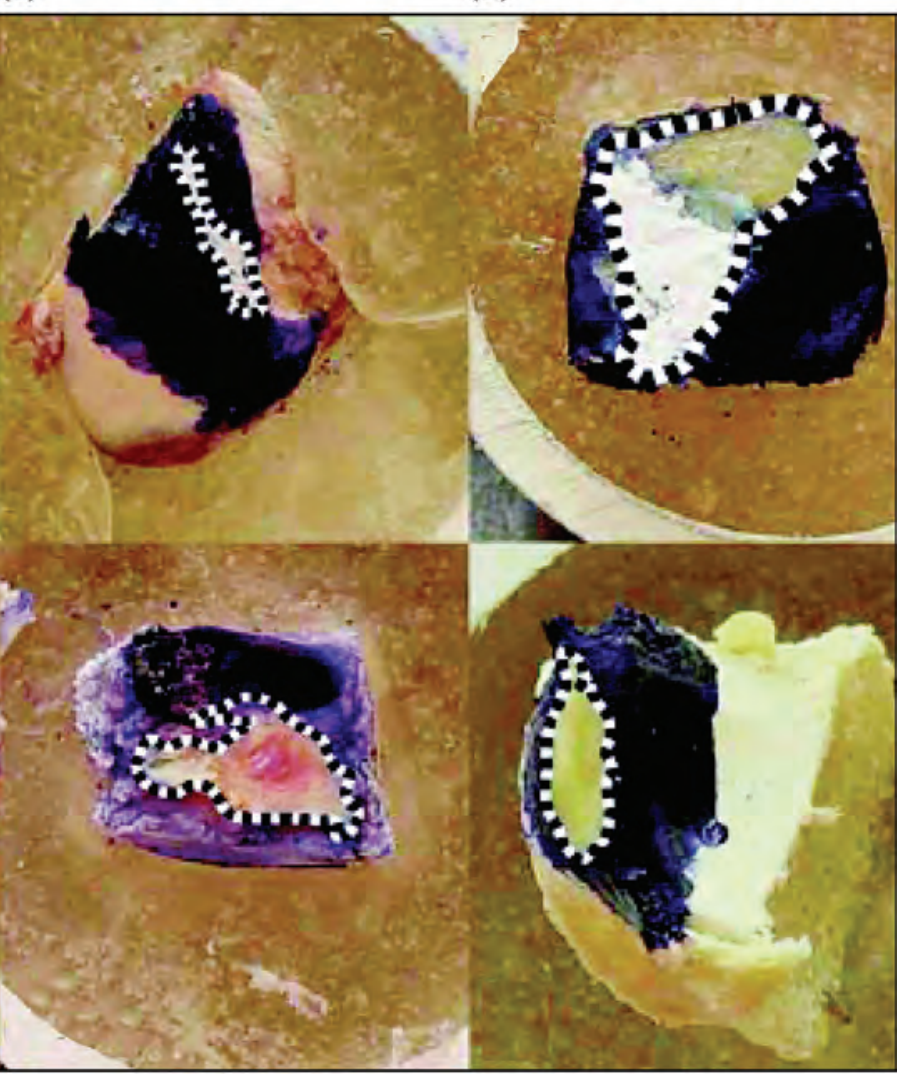

(g) (h)

Figure 5.

Sample ligament images highlighting variety of shapes encountered: (a) TCL, (b) DCbM5, (c) MC1M1, (d) IC3Cb, (e) DC2Nv, (f) IM1M2, (g) IC2C3, and (h) PTTL. Dotted lines delineate ligament cross-sectional shape. See Table 1 for key to ligament acronyms.

\section{DISCUSSION}

We have developed a new method for measuring ligament cross-sectional area to determine foot and ankle ligament morphometry in 121 bone-ligament-bone preparations. The cross-sectional area and length measurements were performed with a digital caliper for length and a freeze-fracture technique for area under identical tare loading conditions $(1.5 \mathrm{~N})$. We observed a variety of complex cross-sectional shapes, many of which are not well suited to other measurement techniques without significant error.

Our ligament morphometry measurements were distinct from data previously reported by Siegler et al. (Figure 7). Overlap spanning in this ankle ligament morphometric spectrum is minimal and exists solely between the ATTL ligament from this study and the PTFL ligament from Siegler et al., as seen in Table 4. The area measurements acquired in this study were larger than those in Siegler et al. This is largely attributable to the mechanisms for measuring crosssectional area. The area micrometer compressed the ligaments with $0.345 \mathrm{MPa}$ of pressure and consistently confined each ligament to a specific shape [8]. The freeze-fracture technique we used had a smaller effect on ligament crosssectional shape and size than the area micrometer.

The length measurements between ligament samples had smaller differences; however, our measurements were consistently longer than Siegler et al.'s. The ligaments measured by Siegler et al. experienced $22 \mathrm{~N}$ of tension, so one would expect to see measurements that were longer than those measured in this study with a $1.5 \mathrm{~N}$ tare load. The difference in measurements was conceivably due to the protocol Siegler et al. used to assess ligament length compared with the insertion-to-origin protocol we used, and to the fact that our sample of feet was of a larger shoe size than that in Siegler et al. Our study population 
Table 2.

Ligament morphometry (mean \pm standard deviation) with use of freeze fracture technique to measure area.

\begin{tabular}{lccc}
\hline Ligament & $\begin{array}{c}\text { Area } \\
\left(\mathbf{m m}^{\mathbf{2}}\right)\end{array}$ & $\begin{array}{c}\text { Length } \\
(\mathbf{m m})\end{array}$ & $\begin{array}{c}\text { Area-to-Length } \\
\text { Ratio }(\mathbf{m m})\end{array}$ \\
\hline IC3Cb & $170.48 \pm 48.10$ & $6.31 \pm 1.79$ & $26.67 \pm 13.29$ \\
IC2C3 & $121.07 \pm 43.97$ & $5.01 \pm 0.97$ & $23.75 \pm 6.75$ \\
IM4M5 & $71.91 \pm 22.94$ & $6.52 \pm 1.21$ & $11.23 \pm 3.66$ \\
ICN & $161.00 \pm 38.91$ & $24.30 \pm 4.78$ & $6.925 \pm 2.715$ \\
DM4M5 & $57.43 \pm 25.44$ & $10.30 \pm 1.41$ & $5.672 \pm 2.749$ \\
DCbM5 & $65.71 \pm 32.04$ & $13.75 \pm 3.81$ & $5.395 \pm 3.390$ \\
DM3M4 & $39.95 \pm 8.073$ & $7.63 \pm 1.37$ & $5.362 \pm 1.375$ \\
PC1M1 & $51.52 \pm 23.66$ & $11.32 \pm 2.06$ & $4.762 \pm 2.501$ \\
IM1M2 & $64.53 \pm 15.54$ & $14.00 \pm 3.34$ & $4.742 \pm 1.305$ \\
DC2M2 & $28.94 \pm 16.08$ & $7.34 \pm 1.71$ & $4.307 \pm 2.914$ \\
DC2Nv & $33.66 \pm 15.59$ & $10.41 \pm 3.28$ & $3.841 \pm 2.836$ \\
ATFL & $62.85 \pm 21.92$ & $18.89 \pm 2.97$ & $3.488 \pm 1.655$ \\
DC1M1 & $28.79 \pm 6.69$ & $9.32 \pm 3.11$ & $3.213 \pm 0.657$ \\
SPL & $98.67 \pm 25.97$ & $30.72 \pm 2.72$ & $3.208 \pm 0.719$ \\
PTTL & $78.43 \pm 39.59$ & $26.68 \pm 4.49$ & $3.045 \pm 1.725$ \\
MC1M1 & $30.90 \pm 16.98$ & $12.03 \pm 2.42$ & $2.824 \pm 2.115$ \\
ATTL & $43.49 \pm 19.92$ & $24.09 \pm 8.03$ & $1.912 \pm 0.756$ \\
PTFL & $46.43 \pm 21.33$ & $27.74 \pm 3.41$ & $1.773 \pm 1.100$ \\
TCL & $43.20 \pm 28.57$ & $37.45 \pm 2.74$ & $1.178 \pm 0.839$ \\
CFL & $21.36 \pm 7.06$ & $35.44 \pm 6.31$ & $0.634 \pm 0.278$ \\
\hline${ }^{*}$ See Table 1 for key to ligament acronyms. & \\
\hline
\end{tabular}

included bone-ligament-bone preparations from 20 adult males. By only including males, we controlled for potential sex-related differences in ligament morphometry. Further, this data set also bracketed the age ( $67 \mathrm{yr}$ ) of the adult male subject we used to develop our 3-D FE model of the lower limb [5-7]. In contrast, the Siegler et al. study measured ligament samples from 12 females and 8 males [8].

Each area measurement technique has its benefits and shortcomings. The simplest method, calculation of crosssectional area from caliper measurements that assumed the ligament was rectangular or ellipsoid in shape, was very repeatable and quickly performed; however, complex shapes with odd concave surfaces tended to cause overestimations of true area, while application of the calipers may have compressed the ligament and altered the measurement of the dimension sought. Race and Amis found that the area micrometer reduced the measured value over time similar to viscoelastic relaxation [16]. Reductions by as much as 25 percent of initial measured cross-sectional area were identified during $600 \mathrm{~s}$ of continual compression, where the

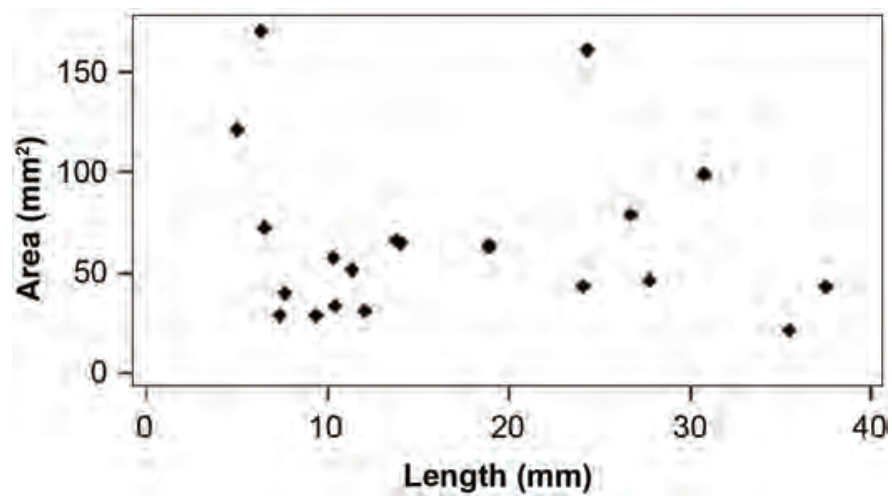

Figure 6.

Morphometric spectrum of mean measurements of 20 male cadaver foot ligament specimens.

120 s time point was identified as 100 percent of crosssectional area. The prevailing theory is that the area micrometer deformed the ligament as water and ground substance flowed away from the compression site [15-16,21].

Woo et al. measured rabbit ACL and MCL with an area micrometer (which applied 0.12 MPa of pressure), a laser micrometer, and digital calipers and noted that the area micrometer and calipers underestimated area compared with the laser micrometer. The calipers tended to "flatten" the ligaments, which resulted in underestimation [15]. Digital calipers have been reported to underestimate area by 2 percent on average compared with the laser micrometer, and the area micrometer underestimated area by 19 percent [15] and 17 percent [21] on average when compared with the laser micrometer. The laser micrometer has been reported to have difficulties with concavities and complex surfaces [15,21], and the knee ligaments of the rabbits tested had "uniquely simple geometries" [15], which were likely more simple than the foot ligaments measured in this study. Woo et al. also reported that the laser micrometer could not be used effectively when the laser path was obscured by short ligaments with bony projections, which are abundant in the foot [15].

The casting method developed by Race and Amis replicates a ligament shape and size by creating ligament molds. The casts were generated with liquid silicon rubber and a curing agent. PMMA was cast in these silicon molds for creation of ligament replicas that were sectioned, stained, and photographed with an in-plane calibration. The investigators measured area by counting pixels of the photographs that were enlarged onto graph paper. The 
Table 3.

Comparison between digital caliper and freeze-fracture (FF) techniques for measurement of ligament cross-sectional area. ${ }^{*} \mathrm{Q} \% \mathrm{Diff}$ represents the \% difference associated with rectangular caliper (Q) approximation based on FF measurements (averaged over each ligament type). E\% Diff represents \% difference associated with ellipsoid caliper (E) approximation based on FF measurements (averaged over each ligament type). Positive Q\% Diff and E\% Diff values represent underestimation of average ligament FF area and negative values represent overestimation.

\begin{tabular}{lrrrrr}
\hline \multirow{2}{*}{ Ligament $^{\dagger}$} & \multirow{2}{*}{ Q\% Diff } & \multirow{2}{*}{ E\% Diff } & \multicolumn{3}{c}{ Area (mm $\mathbf{~}^{2} \mathbf{~}$} \\
\cline { 3 - 5 } & & & $\mathbf{Q}$ & $\mathbf{E}$ & $\mathbf{F F}$ \\
\hline ICN & 29.14 & 44.34 & 114.09 & 89.61 & 28.46 \\
DM4M5 & 36.76 & 50.44 & 36.32 & 26.37 & 57.43 \\
DCbM5 & 48.91 & 59.87 & 33.57 & 25.77 & 39.71 \\
DM3M4 & 17.87 & 35.49 & 32.81 & 29.95 & 51.52 \\
PC1M1 & 25.97 & 41.87 & 38.14 & 38.91 & 64.53 \\
IM1M2 & 23.23 & 39.70 & 49.54 & 14.88 & 28.94 \\
DC2M2 & 34.55 & 48.58 & 18.94 & 20.34 & 33.66 \\
DC2Nv & 23.05 & 39.57 & 25.90 & 31.86 & 62.85 \\
ATFL & 35.47 & 49.31 & 40.56 & 14.66 & 28.79 \\
DC1M1 & 35.19 & 49.08 & 18.66 & 53.03 & 98.67 \\
SPL & 31.57 & 46.26 & 67.52 & 76.90 & 78.43 \\
PTTL & -24.84 & 1.95 & 97.91 & 21.23 & 30.90 \\
MC1M1 & 12.52 & 31.29 & 27.03 & 35.12 & 43.49 \\
ATTL & 19.25 & 19.25 & 44.72 & 41.93 & 46.43 \\
PTFL & -14.99 & 9.69 & 53.39 & 30.19 & 43.20 \\
TCL & 11.02 & 30.12 & 38.44 & 15.80 & 21.36 \\
CFL & 5.81 & 26.03 & 20.12 &
\end{tabular}

*IC3Cb, IC2C3, and IM4M5 ligaments not included because of measurement difficulties with calipers.

${ }^{\dagger}$ See Table 1 for key to ligament acronyms.

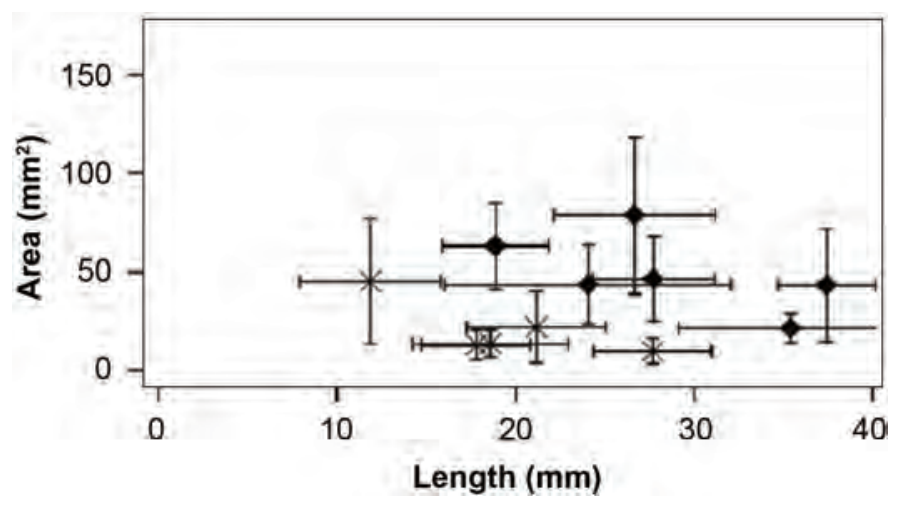

Figure 7.

Comparison of mean ankle measurements from this study $(\bullet)$ vs Siegler et al. $(\times)$ (Source: Siegler S, Block J, Schneck CD. The mechanical characteristics of the collateral ligaments of the human ankle joint. Foot Ankle. 1988;8(5):234-42). Mean ligament values provided with error bars representing 1 standard deviation from sample mean.

casting technique was reported to systematically underestimate cross-sectional area because of shrinkage during the curing process. The area micrometer underestimated the casting technique by 16 percent on average [16]. Esti- mates of the laser micrometer performance were compared with the casting technique when area of the posterioromedial bundles of the PCL, noted for having significant concavities, was measured. The laser micrometer system would have overestimated the casting method by 19 percent on average [16].

The location for ligament bisection was based purely on visual inspection for both the freeze-fracture and digital caliper protocols; we used no specific markers to identify locations for the measurement along the ligament longitudinal axis. The digital caliper width and thickness measurements tended to be smaller than those from the freeze-fracture technique, which indicates that during the measurement process the calipers slightly compressed the ligaments, or less likely, during the freeze-fracture immersion process the ligaments increased in girth. The cross-sectional shape of the ligaments may also have varied across the midportion of each ligament, adding to the potential error of our comparisons. The difference between both methods can be explained by systemic underestimates with the calipers and variation in the measurement sites. 
Table 4.

Comparison between ankle ligament morphometry measurements (mean \pm standard deviation) from this study (freeze-fracture [FF]) vs. Siegler et al. (area micrometer $[\mathrm{AM}])$.

\begin{tabular}{|c|c|c|c|c|}
\hline \multirow{2}{*}{ Ligament $^{\dagger}$} & \multicolumn{2}{|c|}{ Area (mm²) } & \multicolumn{2}{|c|}{ Length (mm) } \\
\hline & FF & AM & FF & $\mathbf{A M}$ \\
\hline ATFL & $62.85 \pm 21.92$ & $12.90 \pm 7.70$ & $18.89 \pm 2.97$ & $17.81 \pm 3.05$ \\
\hline ATTL & $43.49 \pm 19.92$ & - & $24.09 \pm 8.03$ & - \\
\hline PTFL & $46.43 \pm 21.33$ & $21.90 \pm 18.10$ & $27.74 \pm 3.41$ & $21.16 \pm 3.86$ \\
\hline TCL & $43.20 \pm 28.57$ & - & $37.45 \pm 2.74$ & - \\
\hline TNL & 一 & $7.10 \pm 2.60$ & 一 & $41.83 \pm 4.93$ \\
\hline TSL & - & $13.50 \pm 7.10$ & - & $18.59 \pm 4.37$ \\
\hline
\end{tabular}

The range of the area and length morphometry of the ligaments in the foot and ankle is presented in Figure 6. This is the first morphometric spectrum to present how the ligaments within the foot and ankle may be short and thin, short and thick, long and thin, and long and thick. Establishment of this morphometric spectrum is the initial step toward an understanding of the structure-function relationships of foot and ankle ligaments, which are unique from other well-established ligament mechanical properties.

Critical to the development of our and other groups' foot models is the assignment of accurate material properties for the multitude of soft-tissue constituents [1-7]. The lack of published foot and ankle ligament data, coupled with the numerous tests that would be required to obtain a statistical sampling of ligament properties for the 51 ligaments in our model (with more to be added), motivated this current study. Our long-term goal is to verify whether material properties of ligaments can be reasonably approximated from their morphometry alone. Critical to this goal was finding a means to accurately measure ligament length, and more importantly, ligament cross-sectional area. Although beyond the scope of this article, one should note that mechanical property measurements were made for each ligament prior to application of the destructive freeze-fracture technique. Our future work will establish a relationship between the nonlinear material properties and the ligament morphometry. This relationship will be used to interpolate nonlinear material properties for foot ligaments where material properties are unknown but morphometry can be obtained via imaging techniques.

The freeze-fracture technique was prone to the following potential sources of error. With the area meas- urements, we assumed that ligament was the only tissue photographed during the image-acquisition process. Some residual fat possibly remained from the dissection and resulted in overestimation. Crystallized water vapor from the immersion process may have reduced the surface dye contrast and resulted in overestimation. The contrast was improved by application of additional dye to the perimeter and application of Liquid Paper to the ligament cross section (Figure 5(a)-5(d), 5(f)). Shearing generated during the ligament bisection may have resulted in overestimation if the ligament was not completely frozen and the resultant tissue was frayed or smeared. We investigated this by obtaining side-profile images (digital photos) of five randomly selected ligaments prior to bisection. After bisection, no discernible increase in ligament height was observed for any of these ligaments, which supports that the fracturing technique was not introducing additional artifact. Additionally, this method assumes no plastic deformation during mechanical testing.

Furthermore, the freeze-fracture technique is a destructive technique that prohibits further use of the ligament after sectioning (i.e., destructive load-to-failure measurements cannot be performed if one employs our freeze-fracture protocol). The protocol used in this study did not allow for multiple measurements along the ligament length, a feature supported by other area measurement techniques. However, revision of our bisection process by use of another tool similar to those for histologic dissections would allow comparison of areas and shapes along ligament length.

We addressed the accuracy of the digital calipers to determine errors related to ligament length measurements. When checked with a rule graduated to $1 \mathrm{~mm}$, accuracy 
was 99.5 percent when repeated measures were performed. Repeatability was performed on the worst-case scenario of measurement: the smallest expected ligament that was potted in a potting cup. A $4 \mathrm{~mm}$ graduated rule was potted and 20 measurements were performed, where the average measurement was $4.02 \pm 0.14 \mathrm{~mm}$. The coefficient of variation among all the measurements was 3.47 percent about the mean. The resolution of the calipers was $0.01 \mathrm{~mm}$.

\section{CONCLUSIONS}

Foot and ankle ligament morphometry has been established by identification of the morphometric spectrum in cross-sectional area and ligament length. The freezefracture protocol has been established as a technique for measuring foot and ankle ligament cross sections. The freeze-fracture technique is better adapted to measurement of complex cross-sectional geometry of the foot and ankle ligaments when compared with the digital caliper, the area micrometer, and the laser micrometer. The freeze-fracture technique is slightly better at approximating complex area measurements than the casting technique, since the casting technique is prone to a shrinkage effect. A correction factor has been established for use with the casting technique; however, the reported shrinkage for rabbit knee ligaments has not been validated against the variant geometric shapes present in the foot and ankle. In addition, the freeze-fracture technique is the least difficult for estimation of morphometry for short ligaments (less than $10 \mathrm{~mm}$ ) or ligaments with bony projections that would obscure necessary line-of-sight conditions, both of which were abundant in our sample of foot ligaments.

\section{ACKNOWLEDGMENT}

Dr. Chimba Mkandawire now works for Exponent, Inc, in Philadelphia, Pennsylvania, and holds a research appointment with the School of Biomedical Engineering, Science, and Health Systems at Drexel University in Philadelphia, Pennsylvania.

\section{REFERENCES}

1. Chen WP, Tang FT, Ju CW. Stress distribution of the foot during mid-stance to push-off in barefoot gait: a 3-D finite element analysis. Clin Biomech (Bristol, Avon). 2001; 16(7):614-20.

2. Cheung JT, Zhang M, An KN. Effects of plantar fascia stiffness on the biomechanical responses of the ankle-foot complex. Clin Biomech (Bristol, Avon). 2004;19(8):839-46.

3. Gefen A, Megido-Ravid M, Itzchak Y, Arcan M. Biomechanical analysis of the three-dimensional foot structure during gait: a basic tool for clinical applications. J Biomech Eng. 2000;122(6):630-39

4. Salathe EP, Arangio GA. A biomechanical model of the foot: the role of muscles, tendons, and ligaments. J Biomech Eng. 2002;124(3):281-87.

5. Camacho DL, Ledoux WR, Rohr ES, Sangeorzan BJ, Ching RP. A three-dimensional, anatomically detailed foot model: a foundation for a finite element simulation and means of quantifying foot-bone position. J Rehabil Res Dev. 2002;39(3):401-10.

6. Ledoux WR, Camacho DL, Ching RP, Sangeorzan BJ. The development and validation of a computational foot and ankle model. In: Proceedings of the World Congress of Medical Physics and Biomedical Engineering; $2000 \mathrm{Jul}$ 23-28; Chicago, IL.

7. Ledoux WR, Camacho DL, Ching RP, Sangeorzan BJ. A finite element model of the foot and ankle: validation of quiet stance. In: Proceedings of the 4th World Congress of Biomechanics; 2002 Aug 4-9; Calgary, Canada. p. 5605.

8. Siegler S, Block J, Schneck CD. The mechanical characteristics of the collateral ligaments of the human ankle joint. Foot Ankle. 1988;8(5):234-42.

9. Mkandawire C, Ledoux WR, Sangeorzan BJ, Ching RP. Hierarchical cluster analysis of area and length of foot and ankle ligaments. In: Proceedings of the 25th Annual Meeting of the American Society of Biomechanics; 2001 Aug 8-11; San Diego, CA. p. 367-68.

10. Mkandawire C. The relationship between viscoelastic relaxation and ligament morphometry [doctoral dissertation]. Seattle (WA): University of Washington; 2002.

11. Johnson GA, Tramaglini DM, Levine RE, Ohno K, Choi NY, Woo SL. Tensile and viscoelastic properties of human patellar tendon. J Orthop Res. 1994;12(6):796-803.

12. Torzilli PA, Arnoczky SP. Mechanical properties of the lateral collateral ligament: effect of cruciate instability in the rabbit. J Biomech Eng. 1988;110(3):208-12. Erratum in: J Biomech Eng 1988;110(4):299.

13. Danto MI, Woo SL. The mechanical properties of skeletally mature rabbit anterior cruciate ligament and patellar tendon over a range of strain rates. J Orthop Res. 1993; 11(1):58-67.

14. Davis WH, Sobel M, DiCarlo EF, Torzilli PA, Deng X, Geppert MJ, Patel MB, Deland J. Gross, histological, and microvascular anatomy and biomechanical testing of the spring ligament complex. Foot Ankle Int. 1996;17(2):95-102. 
15. Woo SL, Danto MI, Ohland KJ, Lee TQ, Newton PO. The use of a laser micrometer system to determine the crosssectional shape and area of ligaments: a comparative study with two existing methods. J Biomech Eng. 1990;112(4): 426-31.

16. Race A, Amis AA. Cross-sectional area measurement of soft tissue. A new casting method. J Biomech. 1996;29(9): 1207-12.

17. Du T, Xu LJ, Lei M, Wang NS, Eidelman DH, Ghezzo H, Martin JG. Morphometric changes during the early airway response to allergen challenge in the rat. Am Rev Respir Dis. 1992;146(4):1037-41.

18. Sarrafian SK. Anatomy of the foot and ankle: descriptive, topographic, functional. 2nd ed. Philadelphia (PA): Lippincott; 1993.
19. Bettinger PC, Smutz WP, Linscheid RL, Cooney WP 3rd, An KN. Material properties of the trapezial and trapeziometacarpal ligaments. J Hand Surg [Am]. 2000;25(6): 1085-95.

20. Clark JM, Norman AG, Kaab MJ, Notzli HP. The surface contour of articular cartilage in an intact, loaded joint. J Anat. 1999;195(Pt 1):45-56.

21. Lee TQ, Woo SL. A new method for determining crosssectional shape and area of soft tissues. J Biomech Eng. 1988;110(2):110-14.

Submitted for publication August 5, 2004. Accepted in revised form August 9, 2005. 